\title{
Review \\ Effect of Physical Therapy Modalities on Quality of Life of Head and Neck Cancer Survivors: A Systematic Review with Meta-Analysis
}

\author{
Barbara Burgos-Mansilla ${ }^{1}$, Noelia Galiano-Castillo 2,3,4,5 (D), Mario Lozano-Lozano 2,3,4,5,*(D), \\ Carolina Fernández-Lao $2,3,4,5$, Maria Lopez-Garzon $2,3,4,5$ and Manuel Arroyo-Morales ${ }^{2,3,4,5}$
}

1 Kinesiology Program, Faculty of Health Sciences, Universidad Autónoma de Chile, Avenida Alemania, Temuco 4810101, Chile; barbara.burgos@uautonoma.cl

2 Department of Physical Therapy, Faculty of Health Sciences, University of Granada, 18016 Granada, Spain; noeliagaliano@ugr.es (N.G.-C.); carolinafl@ugr.es (C.F.-L.); maloga@ugr.es (M.L.-G.); marroyo@ugr.es (M.A.-M.)

3 Sport and Health University Research Institute (iMUDS), 18016 Granada, Spain

4 Instituto de Investigación Biosanitaria ibs.GRANADA, 18016 Granada, Spain

5 'Cuídate' Support Unit for Oncology Patients (Bio277 Group), 18016 Granada, Spain

* Correspondence: mlozano@ugr.es

Citation: Burgos-Mansilla, B.; Galiano-Castillo, N.; Lozano-Lozano, M.; Fernández-Lao, C.; Lopez-Garzon, M.; Arroyo-Morales, M. Effect of Physical Therapy Modalities on Quality of Life of Head and Neck Cancer Survivors: A Systematic Review with Meta-Analysis. J. Clin. Med. 2021, 10, 4696. https://doi.org/ $10.3390 / \mathrm{jcm} 10204696$

Academic Editor: Alexandre Bozec

Received: 15 September 2021

Accepted: 11 October 2021

Published: 13 October 2021

Corrected: 20 January 2023

Publisher's Note: MDPI stays neutral with regard to jurisdictional claims in published maps and institutional affiliations.

Copyright: (c) 2021 by the authors. Licensee MDPI, Basel, Switzerland. This article is an open access article distributed under the terms and conditions of the Creative Commons Attribution (CC BY) license (https:/ / creativecommons.org/licenses/by/ $4.0 /)$.

\begin{abstract}
The objective was to describe the effectiveness of different physical therapy modalities to improve Quality of Life (QoL) in Head and Neck Cancer (HNC) survivors. PubMed, Scopus, Web of Science, CINAHL and Cochrane Library were searched for randomized clinical controlled trials published until 30 April 2020. Risk of bias assessment and meta-analysis were conducted using the Cochrane tools. A total of 251 records were retrieved, and 10 met the inclusion criteria. Interventions whose parameters focus on a 12-week exercise programs of aerobic activity (walking) or Progressive Resistance Training (PRT) for the whole body are effective and safe modalities improving QoL in HNC survivors. Electrophysical agents did not show significant results between groups. As for the assessment of methodological quality, 4 of the 10 articles included had a high risk of overall bias. Only five articles provided sufficient information to conduct a meta-analysis for exercise program intervention on QoL, showing a tendency in favor of intervention group, even when the global results did not show statistically significant improvements (pooled Cohen's d 0.11; 95\% CI: -0.27 to $0.50 ; \mathrm{I}^{2} 42.68 \%$; $p$ heterogeneity $=0.12$ ). The present review and meta-analysis identified meaningful benefits of exercise on QoL of HNC survivors; this has been confirmed in a meta-analysis. This review adds evidence supporting exercise interventions on Head and Neck Cancer population whose opportunities for successful recovery after medical treatment are more limited.
\end{abstract}

Keywords: systematic review; meta-analysis; head and neck neoplasms; cancer survivors; physical therapy modalities; quality of life

\section{Introduction}

Head and Neck Cancer (HNC) covers sites located on the lips (mucosa surface), oral cavity, pharynx, larynx, cervical esophagus, nose, paranasal sinuses, salivary glands, thyroid gland and parathyroid glands [1]. Both early detection programs and better treatments have been responsible for the steady increase in the survival rate of these patients, in addition to a decrease in smoking habits and better prognosis with human papillomavirus (HPV)-derived cancer $[2,3]$. For instance, $65.3 \%$ of patients diagnosed with HNC in the United States survive 5 years or more [4], and half of people diagnosed with HNC in England survive 10 years or more [5]. HNC mainly affects people of working age, and thus, the economic costs are very high (a 5-year mean cost of USD 79,165 per patient) [6].

A large proportion of these patients receive surgery (32-75\%) [5], radiotherapy (RT; 43-85\%) [5] and/or chemoradiotherapy (CRT; 8-61\%) [5] as part of their primary cancer 
treatment. Surgery, which is determined by the stage and location of the tumor, presents a diversity of effects according to the timeframe performed, that is, before or after other oncology treatments. Unfortunately, there are adverse effects that remain even 10 years after surgery, such as pain and active trigger points on the head, neck and shoulder muscles, general hypersensitivity and hyperalgesia [7], insomnia and eating problems [8]. In addition, the emotional component of body image is a troublesome factor, considering that the location of these tumors makes them more visible [9]. Conventional 3D RT is related to many delayed impairments, such as trismus, dentition breakdown (radiation caries), loss of salivary gland functions and osteoradionecrosis [10]. Fatigue, emotional distress and low quality of life (QoL) have been described as consequences of intensity-modulated RT [11]. Finally, many HNC patients are treated with CRT (e.g., cetuximab since 2006 in combination with RT). Currently, cisplatin is the most frequently used treatment [12]. CRT implies the greatest disability compared to surgery or RT alone. For example, nutrition impact symptoms such as xerostomia, dysphagia, trismus and oral pain have been described $[13,14]$. Consequently, all of these biopsychofunctional impairments, due not only to the illness but also to the treatments, have a significant impact on the general QoL of these patients.

"Cancer survivor" has been defined in different ways; one of them is as follows: patients living beyond the end of treatment or 3-5 years from diagnosis in complete remission $[15,16]$.

The survival rate in $\mathrm{HNC}$ is one of the most complex owing to the anatomical difficulty of this region [17] and the consequences of medical treatment [18]. Moreover, it is a completely neglected population in terms of rehabilitation strategies $[19,20]$ (if compared to other cancer survivors, such as breast or colorectal cancer). This fact could be due to the short follow-up motivated by nearly $80-90 \%$ of all recurrences occurring within the first years. According to Haddad and Limaye [21], there are no data to guide the follow-up of long-term HNC patients, especially head and neck squamous cell carcinoma survivors. The understanding of complications suffered by these patients is based on assessments developed during the first years after treatment completion. That is why there are few and poor studies reporting long-term side effects, which could explain the lack of data on rehabilitation strategies. Specific efforts should be made to design adequate support strategies and rehabilitation programs in this population [22,23].

Physical therapists, who are responsible for examining and managing the side effects derived from cancer and its treatment, have become an indispensable part of the continuum of cancer care [24-26]. Different approaches to physical therapy (rehabilitation) might considerably reduce the economic impact of this disease, improving the chances of returning to work [19]. One of the main, widely recognized indicators would be their decrease in terms of QoL perception relating to unemployment or reduced work hours [27]. According to this, QoL could be the outcome that reflects the barriers and interference with daily life experienced by HNC survivors [20].

Between both published reviews and meta-analyses, there is a tendency to evaluate the effects of exercise [28-36] in cancer survivors. These interventions have mainly been proven in breast [28-31] and colorectal cancer [32,33]. With regard to HNC survivors, electrotherapy such as transcutaneous electrical nerve stimulation (TENS) $[37,38]$ and laser therapy [39] have reported promising results on salivary flow rate and QoL. Even acupuncture has shown only a discrete effect, increasing salivary function in patients after RT [40]. Jaw exercises and the use of oral devices have been shown to be useful for mouth interincisal opening in cancer treatment-induced trismus [41]. There are previous systematic reviews that have assessed different interventions in HNC survivors [42-49]; however, some include HNC patients undergoing active treatment as a target population [44,47,48]. None of the mentioned reviews consider QoL as a primary outcome, and most focus on oral and swallowing impairments [43-48]. Only the systematic review led by Almeida et al. [42] presents results from studies that measure QoL, but as a secondary outcome. However, [42] uses a valid method and describes that its aim was to assess rehabilitation 
interventions as a whole and does not compile all potential methods that could be effective in improving QoL. In fact, it was only focused on the assessment of shoulder function. All reports mentioned above, despite the promising findings, present some limitations already described. This highlights the need for an updated review that comprises all these criteria. Therefore, this systematic review aimed to describe the effectiveness of different physical therapy modalities to improve QoL in HNC survivors and to discover which of these modalities would be most effective.

\section{Materials and Methods}

\subsection{Focused Question}

A systematic review protocol that defined inclusion criteria, search strategy and outcomes of interest was developed and registered with PROSPERO (CRD 42020151929, 12 May 2020). Reporting of this systematic review adheres to the Preferred Reporting Items for Systematic Reviews and Meta-Analyses (PRISMA) statement [50]. According to PRISMA guidelines, the specific question posed for the review was, "Are physical therapy modalities effective in improving QoL in HNC survivors? Which physical therapy modalities are most effective in improving the QoL of these patients?"

\subsection{Search Strategy and Eligibility Criteria}

Detailed search strategies were developed for each database used in the review: Medline (via PubMed searcher), Scopus, Web of Science, Cumulative Index for Nursing and Allied Health Literature (CINAHL) and Cochrane Library. The literature search was conducted between 1 March and 30 April 2020. The following keywords were used for the search: "head and neck cancer", "survivor", "physical therapy modalities", "quality of life" (see supplementary material: search strategy). Keywords were combined using the Boolean operators "AND" and "OR". No restrictions were placed on the year of publication, but only published studies in Spanish and English from inception to 30 April 2020 were considered. Studies were included if they met the following criteria: (1) design: randomized controlled trials (RCTs); (2) population: adults (over 18 years old) considered to be HNC survivors; (3) intervention: physical therapy modalities such as electric stimulation therapy (electroacupuncture, pulsed radiofrequency treatment, transcutaneous electric nerve stimulation), exercise therapy, hydrotherapy, musculoskeletal manipulations (manipulation, motion therapy, massage), myofunctional therapy and laser therapy (low-level light therapy); (4) control group: placebo, usual care or no intervention; and (5) outcome: QoL. Furthermore, an automatic alert notification for new publications relevant to search term combination was created in all databases from the initial search date. Two independent researchers (B.B.M. and M.L.L.) performed the selection of the studies through Covidence systematic review software (Veritas Health Innovation, Melbourne, Australia) [51]; then, the same two reviewers made the final selection of the studies and appraisal of methodological quality. Disagreements were resolved by the judgment of a third author (N.G.C.).

\subsection{Data Extraction and Quality Assessments}

This process was performed independently by two review authors (B.B.M. and M.L.L.) using an Excel spreadsheet applying a predesigned criterion data collection form. The data collected were study characteristics such as authors, country of origin, study design and sample size and participant characteristics such as sex, mean age, stage of cancer (I, II, III or IV), location of HNC (throat, oral, nose, etc.) and type of oncological treatment (radio, chemo, surgery) were included in the data. Additionally, data on the characteristics of the interventions included frequency, duration, comparison, outcome measures, adverse events, measured time points, intervention group (IG), control group (CG), mean change, group differences in mean change and $p$-values. The outcome measures, such as the European Organization for Research and Treatment of Cancer Questionnaire (EORTC QLQ-C30) [52], Functional Assessment of Cancer Therapy General and Head and Neck 
Module (FACT G and FACT H\&N) [53] and Head and Neck Cancer Inventory (HNCI) [54] were recorded. The risk of bias assessment was performed according to the Cochrane Risk of Bias tool: RoB 2 [55].

After the data extraction, the reviewers determined the possibility of performing a meta-analysis by considering if the heterogeneity was moderate or strong as assessed by $\mathrm{I}^{2}$ (less than $25 \%$, no heterogeneity; $25-49 \%$, low heterogeneity; $50-74 \%$, moderate heterogeneity; and $75 \%$ or greater, high heterogeneity) [56]. For the quantitative combination of the studies, only those that measured QoL by means of a validated instrument presented all of the data necessary to perform it and whose intervention was exercise. With the aim of homogenizing the results, a quantitative combination by subgroups was performed according to the questionnaire used to measure QoL (EORTC QLQ-C30, FACT H\&N, FACT $\mathrm{G})$, and forest plots were used to summarize the results. The studies were combined using the random-effects model of the DerSimonian and Laird method, which considers the variations within and between studies, using Cohen's d effect size as an estimator. The random effects model was used for the analysis. Given the number of included articles (less than 10), it was not possible to perform the publication bias study. For all of the analyses, Stata Statistical Software was used (StataCorp. 2019. Stata Statistical Software: Release 16. StataCorp LLC, College Station, TX, USA).

\section{Results}

The literature search identified 251 articles, with 77 duplicates, and automatic alert notification provided information on approximately 1 new article, which was also included. A total of 148 articles were excluded after screening the titles and abstracts. After that, 27 studies were then retrieved for full-text review, and 17 records were excluded for the following reasons: 3 had incorrect patient populations, 9 were not RCTs, 2 had people undergoing active treatment, 1 included patients with metastasis, 1 included other treatments (nonsteroid anti-inflammatory drugs-NSAIDs) and 1 did not include QoL as a relevant outcome measure. Finally, 10 records were included in this systematic review. Interrater agreement in the selection of studies was 51.4\% [57]. After discussion, the reviewers reached consensus $(100 \%)$. In the PRISMA flowchart, the stages of the review process, including study identification, inclusion and exclusion, are shown (Figure 1).

\subsection{Descriptive Synthesis}

The 10 studies included in this review were conducted across six countries, most commonly in Canada $(n=4)$ [58-61] and in second place China $(n=2)[62,63]$. A total of 533 subjects participated in the studies included in this review (292 IG and 241 CG), and most were males $(77 \%)$. The sample size of the studies ranged between 20 and 170 subjects. The global mean age of all subjects (IG and CG) was 56.4 years, with a range between 48 and 66 years. Analyzing all participants of the included studies, $26.5 \%$ were in stage I-II at diagnosis, and $73.5 \%$ were in stage III-IV. The most common location of HNC was the pharynx (41\%), followed by other sites and the oral cavity. Of the oncological treatments, the most common was surgery and CRT $(28 \%)$, followed by RT $(20 \%)$; surgery plus RT was the least common (14\%) (Table 1). 
Table 1. Descriptive Synthesis of the included studies.

\begin{tabular}{|c|c|c|c|c|c|c|c|c|c|c|c|c|c|c|c|c|c|c|c|}
\hline \multirow{2}{*}{ Authors } & \multirow{2}{*}{ Country } & \multirow{2}{*}{$\begin{array}{l}\text { Study } \\
\text { Design }\end{array}$} & \multirow{2}{*}{$\begin{array}{c}\text { Sample } \\
\text { Size }\end{array}$} & \multirow{2}{*}{$\begin{array}{l}\begin{array}{c}\text { Gender } \\
(\% \text { Male })\end{array} \\
\text { (1) }\end{array}$} & \multirow{2}{*}{$\begin{array}{c}\text { Mean Age } \\
\text { (Years) }\end{array}$} & \multicolumn{2}{|c|}{$\begin{array}{c}\text { Stage of Cancer at } \\
\text { Diagnosis }(\%)\end{array}$} & \multicolumn{5}{|c|}{ Location of HNC (\%) } & \multicolumn{7}{|c|}{ Oncological Treatment $(\%)$} \\
\hline & & & & & & $\mathrm{I}-\mathrm{II}$ & III-IV & Pharynx & Larynx & Oral Cavity & Lip & Others & RT & CT & $\mathrm{s}$ & CRT & $\mathrm{S}+\mathrm{RT}$ & $\mathrm{S}+\mathrm{CT}$ & $\mathrm{RT}+\mathrm{S}+\mathrm{CT}$ \\
\hline $\begin{array}{l}\text { Chang et al, } 2020 \\
\text { Langmore et al, } 2016\end{array}$ & $\begin{array}{c}\text { China } \\
\text { United States }\end{array}$ & $\begin{array}{l}\mathrm{RCT} \\
\mathrm{RCT}\end{array}$ & ${ }_{170}^{88}$ & $\begin{array}{l}91 \% \\
86 \%\end{array}$ & ${ }^{56}{ }_{61.9}$ & ${ }^{*}{ }_{15}$ & ${ }^{*} 85$ & ${ }^{20}{ }_{70}$ & ${ }_{12}$ & 0 & ${ }^{0}$ & $\begin{array}{ll}80 & \\
80\end{array}$ & ${ }_{29}$ & $\begin{array}{ll}0 & 0 \\
& 0\end{array}$ & ${ }^{100}{ }_{0}$ & $\begin{array}{ll}0 & \\
& 71\end{array}$ & ${ }^{0}$ & 0 & 0 \\
\hline $\begin{array}{l}\text { Lavigne et al., } 20200 \\
\text { Lombro etal } 2013\end{array}$ & $\begin{array}{l}\text { Canada } \\
\text { Denmark }\end{array}$ & ${ }_{\mathrm{RCT}}^{\mathrm{RCT}}$ & $\frac{22}{44}$ & $\begin{array}{l}64 \% \\
66 \%\end{array}$ & $\begin{array}{l}52 \\
57\end{array}$ & 45 & 55 & $\begin{array}{l}59 \\
77\end{array}$ & 5 & 18 & 0 & 18 & 0 & 82 & 18 & 0 & 0 & 0 & ( \\
\hline McNeely et al, 2008 & Canada & RCT & 52 & $71 \%$ & 52 & 18 & 82 & 62 & 23 & 0 & 0 & 15 & 0 & 0 & 0 & 0 & 75 & 25 & ( \\
\hline $\begin{array}{l}\text { O'Neill et al., } 2018 \\
\text { Saleh he al.,2014 }\end{array}$ & $\begin{array}{l}\text { Ireland } \\
\text { Brazil }\end{array}$ & $\begin{array}{l}\text { RCT } \\
\text { RCT }\end{array}$ & $\begin{array}{l}43 \\
23\end{array}$ & $\begin{array}{l}81 \% \% \\
65 \%\end{array}$ & $\begin{array}{l}66 \\
57\end{array}$ & $\begin{array}{l}49 \\
17\end{array}$ & $\begin{array}{l}51 \\
83\end{array}$ & $\stackrel{0}{*}$ & $\stackrel{0}{*}$ & $\stackrel{0}{*}$ & * & ${ }_{*}^{100}$ & $\begin{array}{l}0 \\
0\end{array}$ & $\begin{array}{l}0 \\
0\end{array}$ & $\begin{array}{r}100 \\
0\end{array}$ & $\begin{array}{r}0 \\
26\end{array}$ & $\begin{array}{c}0 \\
43\end{array}$ & $\begin{array}{l}0 \\
0\end{array}$ & 36 \\
\hline $\begin{array}{l}\text { Saleh et al., } 2014 \\
\text { Su et al, } 2017\end{array}$ & China & RCT & 37 & $\begin{array}{l}951 \% \\
922 \%\end{array}$ & 48 & 30 & $\begin{array}{l}60 \\
70\end{array}$ & 0 & 0 & 81 & 0 & 19 & 30 & 43 & $\begin{array}{l}0 \\
27\end{array}$ & $\begin{array}{r}26 \\
0\end{array}$ & $\begin{array}{l}43 \\
30\end{array}$ & $\begin{array}{r}0 \\
43\end{array}$ & 3 \\
\hline Wong et al., 2003 & Canada & RCT & 37 & $75 \%$ & 59 & & & & & & & & 100 & 0 & 0 & 0 & 0 & 0 & ( \\
\hline
\end{tabular}

HNC: Head and Neck Cancer, RT: radiotherapy, CT: chemotherapy, S: surgery, CRT: chemoradiotherapy, RCT: randomized controlled trial, *: not reported.
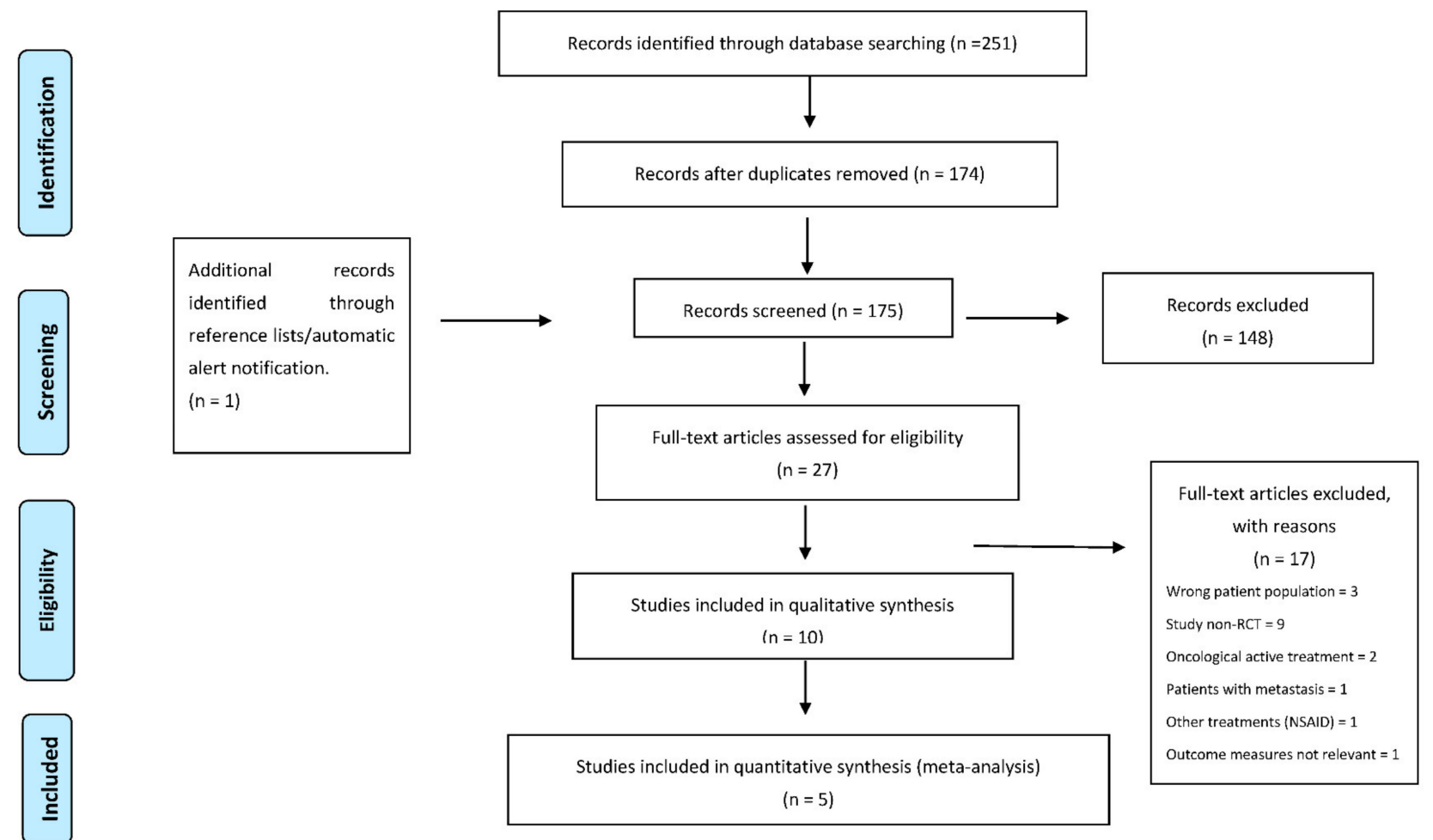

Figure 1. Flowchart of search results and filtering of the documents selected in this study. 
Regarding physical therapy modalities, $60 \%$ of the studies focused on exercise programs [58,59,62-65], and $40 \%$ of the studies were based on electrophysical agents $[60,61,66,67]$. Regarding parameters, there was heterogenicity in terms of type of therapy, frequency and global duration (time per session) for both modalities. Considering exercise, three approaches can be distinguished: first, programs based on aerobic exercise [62]; second, programs based on Progressive Resistance Training (PRT) [58,59,64]; and third, a combination of both $[63,65]$. Regarding parameters for aerobic exercise, one study applied a home-based walking exercise program at a moderate intensity level, $3-5$ days per week for 12 weeks for $30 \mathrm{~min}$ each session, or a total of $150 \mathrm{~min}$ per week [62]. The other two studies $[63,65]$ applied aerobic exercise on a multimodal program through walking with similar parameters. In PRT programs, 2 sets of 8 to 10 repetitions of each exercise $[58,64]$ for 12 weeks were the most commonly used [58,59,63-65]. For the muscle groups involved, three studies focused on upper limb and scapular muscle exercises $[58,59,63]$, and the rest focused on the whole body $[64,65]$.

Considering the electrophysical agents, electrostimulation was the main agent performed $[60,61,67]$. It is important to consider that the aim of electrostimulation differs substantially between studies. One study applied acupuncture-like transcutaneous electrical nerve stimulation (ALTENS) to acupuncture points to improve the salivary flow rate [61]. Two studies used neuromuscular application, one on quadriceps group muscles [60] and the other on suprahyoid muscles [67]. Regarding the parameters of electrostimulation, some similarities were found, with burst modality ranging from $40-70 \mathrm{~Hz}$ as the frequency and a width pulse between 80-300 $\mu \mathrm{s}$. The contraction time varied from 4 to $5 \mathrm{~s}$, a relaxation time of 10-12 s, ramp up 1.5-2 s, ramp down 0-0.75 s and a duration of $20 \mathrm{~min}$. Two out of three studies chose a duration of 12 weeks of intervention $[60,67]$, while the other study used a 6-week intervention time [61]. Only one of four studies applied photobiomodulation therapy (laser therapy) with the following parameters [66]: continuous wave mode, with $830 \mathrm{~nm}$ (infrared) wavelength, $100 \mathrm{~mW}$ output power, $3.57 \mathrm{~W} / \mathrm{cm}^{2}$ power density, $71 \mathrm{~J} / \mathrm{cm}^{2}$ dose per point and application time $20 \mathrm{~s}$ on major salivary glands, parotid glands, submandibular glands and sublingual glands $\left(0.028 \mathrm{~cm}^{2}\right)$. Patients underwent two weekly sessions for 6 weeks. Of the included studies, only three considered follow-up periods [61,62,65], including 12 weeks to 1 year of follow-up. Regarding CG, one study considered placebo treatment [66], another study used placebo and exercises [67], seven studies applied either usual care or education programs [58-60,62-65], and one study contained three groups of interventions [61]. Finally, QoL was measured with different instruments: FACT G/FACT H\&N [58-60,63], EORTC QLQ-C30 [62,64,65] and others, such as the Oral Health Impact Profile (OHIP-14) [66], H\&N Cancer Inventory [67] and Head and Neck Radiotherapy Questionnaire [61] (Table 2). 
Table 2. Summary of the interventions on the included studies.

\begin{tabular}{|c|c|c|c|c|c|c|c|c|c|c|c|c|c|}
\hline \multicolumn{14}{|c|}{ Exercise } \\
\hline Author & Sample Size & Intervention & Frequency & Duration & Comparison & $\begin{array}{l}\text { Outcome } \\
\text { Measures }\end{array}$ & $\begin{array}{c}\text { Adverse } \\
\text { Events }\end{array}$ & $\begin{array}{c}\text { Measured Time } \\
\text { Points }\end{array}$ & $\begin{array}{l}\text { IG (Mean, } \\
\text { SD) }\end{array}$ & CG (Mean, SD) & $\begin{array}{l}\text { Mean Change } \\
\text { (Mean, SD) }\end{array}$ & $\begin{array}{c}\text { Group } \\
\text { Differences in } \\
\text { Mean Change: } \\
\text { Mean ( } 95 \% \mathrm{CI}) \\
\end{array}$ & $p$-Values \\
\hline Chang et al., 2020 & $\begin{array}{l}44 \mathrm{IG} \\
44 \mathrm{CG}\end{array}$ & $\begin{array}{l}\text { Walking exercise and nursing } \\
\text { education health informatics } \\
\text { program }\end{array}$ & $\begin{array}{l}\text { 3-5 days per week } \\
\text { for } 30 \text { min each time, } \\
\text { or a total of } 150 \text { in } \\
\text { per week (from } 55 \% \\
\text { to } 65 \% \text { of HRR) }\end{array}$ & 12 weeks & Usual Care & $\begin{array}{l}\text { EORTC } \\
\text { QLQ-C30 }\end{array}$ & Not reported & $\begin{array}{l}\text { Baseline, week } 4, \\
\text { week 12,3-month } \\
\text { follow-up }\end{array}$ & $\begin{array}{l}\text { Baseline: } 47.5 \\
(5.64)\end{array}$ & $\begin{array}{l}\text { Baseline: } 44.5 \\
(8.50)\end{array}$ & Not reported & Not reported & $\begin{array}{l}\text { Within groups } \\
\text { Baseline-week 4: } \\
\text { IG and CG } p<0.001 \\
\text { Baseline-week 12: } \\
\text { IG and CG } p<0.001 \\
\text { Baseline-3-month } \\
\text { follow-up: } \\
\text { IG and CG } p<0.001 \\
\text { Between groups (IG vs. } \\
\text { CG) } \\
\text { Week 4: } \\
\text { IG }>\text { CG } p<0.05\end{array}$ \\
\hline Lønbro et al., 2013 & $\begin{array}{l}20 \mathrm{IG} \\
21 \mathrm{CG}\end{array}$ & $\begin{array}{l}\text { PRT: leg press, knee extension, } \\
\text { hamstring curls, chest press, } \\
\text { situps, back extensions and } \\
\text { lateral pull down. }\end{array}$ & $\begin{array}{l}2-3 \text { sets of } 8-15 \mathrm{RM} \\
\text { of 7 xercises. } \\
2 \text { to } 3 \text { essions per } \\
\text { week. } \\
\text { total } 30 \text { sessions }\end{array}$ & 12 weeks & Usual Care & $\begin{array}{l}\text { EORTC } \\
\text { QLQ-C30 }\end{array}$ & No & $\begin{array}{l}\text { Baseline and } \\
\text { week 12 }\end{array}$ & $\begin{array}{l}\text { Baseline: } \\
53 \pm 19 \\
\text { Weet } 12: \\
74 \pm 20\end{array}$ & $\begin{array}{l}\text { Baseline: } 70 \pm 15 \\
\text { Week 12: } 78 \pm 18\end{array}$ & $\begin{array}{l}\text { Baseline- } \\
\text { week } 12: \\
\text { IG: } 19 \pm 14 / / \\
\text { CG: } 6 \pm 12\end{array}$ & Not reported & $\begin{array}{l}\text { Withing groups } \\
\text { Baseline-week 12: } \\
\text { II } p<0.001 / \text { / } p ~ p<0.05 \\
\text { Between groups (IG vs. } \\
\text { CG) } \\
\text { Baseline-week 12: } \\
\text { IG }>\text { C } p<0.05 \\
\text { Within groups }\end{array}$ \\
\hline McNeely et al., 2004 & $\begin{array}{l}10 \mathrm{IG} \\
10 \mathrm{CG}\end{array}$ & $\begin{array}{l}\text { PRT on upper limbs and } \\
\text { scapular muscles }\end{array}$ & $\begin{array}{l}1-2 \text { sets of } 15 \text { to } \\
25 \text { RM of } 6 \text { exercises. } \\
3 \text { times per week }\end{array}$ & 12 weeks & Usual Care & FACT H\&N & No & $\begin{array}{l}\text { Baseline and } \\
\text { week 12 }\end{array}$ & $\begin{array}{l}\text { Baseline: } \\
\text { 109.5 (12.2) } \\
\text { Week 12: 104.8 } \\
\text { (18.5) }\end{array}$ & $\begin{array}{l}\text { Baseline: } 103.1 \\
(22.4) \\
\text { Week 12: } 100.9 \\
(23.9)\end{array}$ & $\begin{array}{l}\text { CG: }-2.2 \\
\text { (11.4) } \\
\text { GG: }-4.6(9.0)\end{array}$ & $\frac{-2.4(-13.2 \text { to }}{8.3)}$ & $\begin{array}{l}\text { Baseline-week 12: } \\
\text { IG and CG } p>0.05 \\
\text { Between groups (IG vs. } \\
\text { CG) } \\
\text { Baseline-week 12: } \\
p=0.639\end{array}$ \\
\hline McNeely et al., 2008 & $\begin{array}{l}27 \mathrm{IG} \\
25 \mathrm{CG}\end{array}$ & $\begin{array}{l}\text { PRT on upper limbs and } \\
\text { scapular muscles }\end{array}$ & $\begin{array}{l}2 \text { sets of } 10 \text { to } \\
15 \text { repetitions of } 5 \text { to } \\
8 \text { exercises. } \\
\text { Between } 25 \% \text { at } \\
\text { initial and } 70 \% \text { at the } \\
\text { end of the program } \\
\text { of the } 1-\mathrm{RM}, 3 \text { times } \\
\text { per week } \\
\text { Aerobic: } 3 \text { to } 5 \text { days }\end{array}$ & 12 weeks & Usual Care & FACT G & Pain & $\begin{array}{l}\text { Baseline and } \\
\text { week } 12\end{array}$ & $\begin{array}{l}\text { Baseline: } 79.4 \\
\text { (13.7) } \\
\text { Week 12: } 83.9 \\
\text { (15.6) }\end{array}$ & $\begin{array}{l}\text { Baseline: } 76.4 \\
\text { (18.4) } \\
\text { Week 12: } 78.1 \\
\text { (19.3) }\end{array}$ & $\begin{array}{l}\text { CG: }+1.7(6.9) \\
\text { IG: }+4.4(10.6)\end{array}$ & $+4.5(-0.7$ to 9.7$)$ & $\begin{array}{l}\text { Within groups } \\
\text { Not reported } \\
\text { Between groups (IG vs. } \\
\text { CG) } \\
\text { Baseline--week 12: } \\
p=0.091\end{array}$ \\
\hline O'Neill et al., 2018 & $\begin{array}{l}21 \mathrm{IG} \\
22 \mathrm{CG}\end{array}$ & $\begin{array}{l}\text { Aerobicic walking, stationary } \\
\text { cycling and cross trainining } \\
\text { PRT: upper and lower limb } \\
\text { muscles }\end{array}$ & $\begin{array}{l}\text { per week (from } 30 \% \\
\text { of HRR at initial } \\
\text { weeks to } 60 \% \text { of } \\
\text { HRR at the end of } \\
\text { the programm?RT: } \\
\text { twice a week (from } 2 \\
\text { sets/muscle groups } \\
\text { at initial weeks to } 6 \\
\text { sets / muscle groups } \\
\text { at the end of the } \\
\text { program }\end{array}$ & 12 weeks & Usual Care & $\begin{array}{l}\text { EORTC } \\
\text { QLQ-C30 }\end{array}$ & No & $\begin{array}{l}\text { Baseline, } \\
\text { immediately } \\
\text { postintervention } \\
\text { and at 3-month } \\
\text { follow-up }\end{array}$ & $\begin{array}{l}\text { Baseline: } \\
\text { 75.00 (20.83) } \\
\text { Week 12: 83.33 } \\
\text { (20.83) } \\
\text { 3-month } \\
\text { follow up: } \\
\text { 79.17 (29.16) }\end{array}$ & $\begin{array}{l}\text { Baseline: } 66.67 \\
\text { (33.33) } \\
\text { Week 12: } 66.67 \\
\text { (25.00) } \\
\text { 3-month follow } \\
\text { up: } 75.00 \text { (16.6) }\end{array}$ & Not reported & Not reported & $\begin{array}{l}\text { Within groups } \\
\text { Not reported } \\
\text { Between groups (IG vs. } \\
\text { CG) } \\
\text { Baseline-week 12: } \\
p=0.433 \\
\text { Baseline-3-month } \\
\text { follow-up: } \\
p=0.887\end{array}$ \\
\hline Su et al., 2017 & $18 \mathrm{IG} / 19 \mathrm{CG}$ & $\begin{array}{l}\text { HBP } \\
\text { Aeroic: walking } \\
\text { PRT: upper limb muscles }\end{array}$ & $\begin{array}{l}\text { Once a day } / 5 \text { days } \\
\text { per week } \\
\text { Aerobic: } 50 \text { min } \\
\text { PRT: } 2 \text { sets } / 10 \text { repeti- } \\
\text { tions } / \text { muscle } \\
\text { group }\end{array}$ & $\begin{array}{l}12 \text { consecutive } \\
\text { weeks }\end{array}$ & $\begin{array}{l}\text { OPT: } \\
\text { Aerobic: } \\
\text { Walking was } \\
\text { performed on } \\
\text { treadmill. } \\
\text { No PRT }\end{array}$ & FACT H\&N & Not reported & $\begin{array}{l}\text { Baseline, week } 6 \text {, } \\
\text { week } 12\end{array}$ & $\begin{array}{l}\text { Baseline: } \\
\text { 93.83 (19.73) } \\
\text { Week 6: 94.89 } \\
\text { (22.44) } \\
\text { Week 12: } 93.61 \\
\text { (21.487) }\end{array}$ & $\begin{array}{l}\text { Baseline: } 91.63 \\
\text { (23.59) } \\
\text { Week 6: } 95.21 \\
\text { (22.27) } \\
\text { Week 12: } 103.42 \\
\text { (20.02) }\end{array}$ & Not reported & Not reported & $\begin{array}{l}\text { Within groups } \\
\text { Baseline-week 12: } \\
\text { IG and CG } p>0.05 \\
\text { Between groups (IG vs. } \\
\text { CG) } \\
\text { Baseline-week 12: } \\
p=0.074\end{array}$ \\
\hline
\end{tabular}


Table 2. Cont.

\begin{tabular}{|c|c|c|c|c|c|c|c|c|c|c|c|c|c|c|}
\hline \multicolumn{15}{|c|}{ Electrophysical Agent } \\
\hline Author & Sample Size & Intervention & Parameters & Frequency & Time & Comparison & $\begin{array}{l}\text { Outcome } \\
\text { Measures }\end{array}$ & $\begin{array}{l}\text { Adverse } \\
\text { Events }\end{array}$ & $\begin{array}{l}\text { Measured Time } \\
\text { Points }\end{array}$ & $\begin{array}{l}\text { QoL IG } \\
\text { (Mean, SD) }\end{array}$ & $\begin{array}{l}\text { QoL CG (Mean, } \\
\text { SD) }\end{array}$ & $\begin{array}{l}\text { Mean Change } \\
\text { (Mean, SD) }\end{array}$ & $\begin{array}{l}\text { Group } \\
\text { Differences in } \\
\text { Mean Change: } \\
\text { Mean }(95 \% \text { CI) }\end{array}$ & $p$-Values \\
\hline $\begin{array}{l}\text { Wong } \\
\text { et al., } 2003\end{array}$ & $\begin{array}{l}\text { Group A: } 13 \\
\text { Group B: } 10 \\
\text { Group C: } 14\end{array}$ & $\begin{array}{l}\text { ALTENS } \\
\text { Group A: Sp6, St36, } \\
\text { LII (active and } \\
\text { elctrodes) and } \\
\text { CV24 (indifferent } \\
\text { electrode) }\end{array}$ & $\begin{array}{l}\text { Nonpolarizing, balanced, } \\
\text { biphasicis suare electrical } \\
\text { pulses of } 250-\text { ms. } \\
\text { Trains with a repetition rate of } \\
4 \text { Hz. } \\
\text { Each acupuncture point was } \\
\text { randomly stimulated for } 10 \mathrm{~s} \\
\text { each time }\end{array}$ & Twice weekly & 6 weeks & $\begin{array}{l}\text { ALTENS } \\
\text { Group B: Sp6, } \\
\text { St36, P6 } \\
\text { (active } \\
\text { electrodes) } \\
\text { and CV24 } \\
\text { (indifferent } \\
\text { electrode) } \\
\text { Group C: Sp6, } \\
\text { St5 and 6, P6 } \\
\text { (active } \\
\text { electrodes) } \\
\text { and CV24 } \\
\text { (indifferent } \\
\text { electrode) }\end{array}$ & $\begin{array}{l}\text { Head and } \\
\text { Neck } \\
\text { Radiotherapy } \\
\text { Questionnaire }\end{array}$ & No & $\begin{array}{l}\text { Baseline and } 6,8 \\
\text { and } 12 \text { weeks } \\
\text { after treatment } \\
\text { began and at } 3,6 \\
\text { and } 12 \text { months } \\
\text { after treatment } \\
\text { completion. }\end{array}$ & Not reported & Not reported & Not reported & Not reported & $\begin{array}{l}\text { Within groups } \\
\text { Not reported } \\
\text { Between groups (IG vs. } \\
\text { CG) } \\
\text { Baseline-6 } 6 \text { month } \\
\text { follow-up: } \\
p>0.05\end{array}$ \\
\hline $\begin{array}{l}\text { Saleh et al., } \\
2014\end{array}$ & $12 \mathrm{IG} / 11 \mathrm{CG}$ & $\begin{array}{l}\text { Laser therapy } \\
\text { Application on } \\
\text { major sailivary } \\
\text { glands, parotid, } \\
\text { submandibular and } \\
\text { sublingual glands }\end{array}$ & $\begin{array}{l}\text { Continuous wave mode. } \\
830 \mathrm{~nm} \text { (infrared) wavelength, } \\
100 \mathrm{~mW} \text { output power, } \\
3.57 \mathrm{~W} / \mathrm{cm}^{2} \text { power density, } \\
71 \mathrm{~J} / \mathrm{cm}^{2} \text { dose per point, } 2 \mathrm{~J} \\
\text { energy per point, application } \\
\text { time } 20 \text { sec per point and } 28 \mathrm{~J} \\
\text { dose per session. The area of } \\
\text { the spot was } 0.028 \mathrm{~cm}^{2}\end{array}$ & Twice a week & 6 weeks & $\begin{array}{l}\text { Sham laser } \\
\text { therapy }\end{array}$ & OHIP-14 & Not reported & $\begin{array}{l}\text { Baseline, 6th } \\
\text { session, 12th } \\
\text { session }\end{array}$ & $\begin{array}{l}\text { Baseline: } 10.48 \\
\text { (6.82-14.00) } \\
\text { 6th session: } \\
7.55 \\
\text { (5.65-11.19) } \\
\text { 12th session: } \\
2.5(1.69-9.84)\end{array}$ & $\begin{array}{l}\text { Baseline:10.23 } \\
\text { (6.39-12.82) } \\
\text { 6th session: } 5.17 \\
\text { (2.28-10.69) } \\
\text { 12th session: } 3.53 \\
(0.66-10.44)\end{array}$ & Not reported & Not reported & $\begin{array}{l}\text { Within groups } \\
\text { Baseline-12th session } \\
\text { IG and CG } p<0.05 \\
\text { Between groups (IG vs. } \\
\text { CG) } \\
\text { Baseline, } p=0.786 \\
\text { 6th session } p=0.413 \\
\text { 12th session, } p=0.976\end{array}$ \\
\hline $\begin{array}{l}\text { Langmore } \\
\text { et al, } 2016\end{array}$ & $116 \mathrm{IG} / 54 \mathrm{CG}$ & $\begin{array}{l}\text { E-stim device: } \\
\text { Electrical } \\
\text { Stimulation to } \\
\text { stimulate the } \\
\text { suprahyouid muscles } \\
\text { + swallow excrises. } \\
\text { 5-minute warmup } \\
\text { stretching protocol } \\
\text { followed by } \\
\text { swallowing } \\
\text { 60 times in } \\
\text { synchrony with the } \\
\text { stimulation }\end{array}$ & $\begin{array}{l}\text { Frequency } 70 \mathrm{~Hz} \\
\text { Pulse width } 300 \text { microseconds } \\
\text { (range, } 130-300 \text { ) } \\
\text { Contraction } 4 \mathrm{~s} \text { (range, } 4-8 \text { ) } \\
\text { Relaxation } 12 \mathrm{~s} \text { (range, } 12-16 \text { ) } \\
\text { Ramp up } 2 \mathrm{~s} \text { (range, 2-4) } \\
\text { Ramp down } 0 \mathrm{~s} \\
\text { Amplitude limit } 0-99 \\
\text { Treatment time } 20 \text { min or } \\
\text { longer if needed }\end{array}$ & $\begin{array}{l}\text { Twice per day, } 6 \\
\text { days per week }\end{array}$ & 12 weeks & $\begin{array}{l}\text { Sham device+ } \\
\text { swallowice } \\
\text { execrises. } \\
\text { 5-minute } \\
\text { warmup } \\
\text { strething } \\
\text { protocol } \\
\text { followed by } \\
\text { swallowing } \\
6 \text { times in } \\
\text { synchrony } \\
\text { with he } \\
\text { stimulation }\end{array}$ & HNCI & Not reported & $\begin{array}{l}\text { Week } 7 \text { and week } \\
12\end{array}$ & $\begin{array}{l}\text { Baseline: } \\
32.54(21.04) \\
\text { Week 12: } 38.85 \\
(23.97)\end{array}$ & $\begin{array}{l}\text { Baseline: } 24.18 \\
\text { (18.58) } \\
\text { Week 12: } 30.93 \\
(20.46)\end{array}$ & $\begin{array}{l}\text { IG: } 6.31(17.92) \\
\text { CG: } 6.74 \\
(15.59)\end{array}$ & 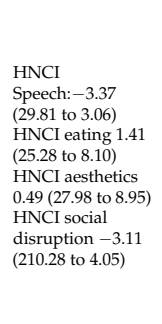 & 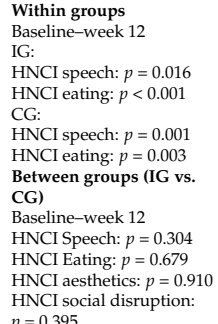 \\
\hline $\begin{array}{l}\text { Lavigne } \\
\text { et al al, } 2020\end{array}$ & $11 \mathrm{IG} / 11 \mathrm{CG}$ & $\begin{array}{l}\text { NMES and } \\
\text { eccentrically } \\
\text { overloaded } \\
\text { unilateral squats }\end{array}$ & 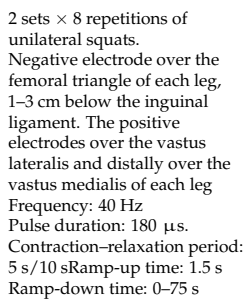 & $\begin{array}{l}\text { Three times per } \\
\text { week }\end{array}$ & 12 weeks & $\begin{array}{l}\text { Conventional } \\
\text { strength } \\
\text { training }\end{array}$ & FACT H\&N & No & $\begin{array}{l}\text { Baseline and } \\
\text { week } 12\end{array}$ & $\begin{array}{l}\text { Baseline: } 116 \\
\text { (18) } \\
\text { Week 12: } 126 \\
\text { (14) }\end{array}$ & $\begin{array}{l}\text { Baseline: } 103(17) \\
\text { Week 12: } 122(13)\end{array}$ & $\begin{array}{l}\text { IG: } 10(9) \\
\text { CG: } 18(9)\end{array}$ & Not reported & $\begin{array}{l}\text { Within groups } \\
\text { Baseline-week } 12 \\
\text { IG and CG } p=0.001 \\
\text { Between groups (IG vs. } \\
\text { CG) } \\
\text { Baseline-week } 12 \\
p>0.05\end{array}$ \\
\hline
\end{tabular}

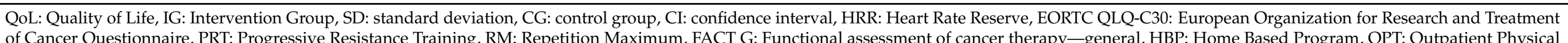

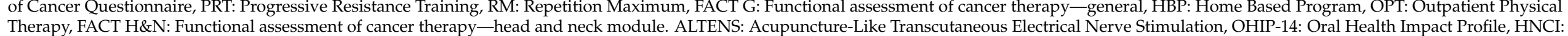
Head and Neck Cancer Inventory, NMES: neuromuscular electrical stimulation. 


\subsection{Adverse Events}

Only one study [58] (10\%) reported adverse events in one subject of the IG who experienced pain related to a soft tissue injury on the scapular region. Fifty percent reported no adverse events [59-61,64,65], and forty percent $[62,63,66,67]$ did not mention adverse events.

\subsection{Qualitative Analysis}

The analysis was performed by subgroups considering each physical therapy modality. Chang et al. [62] and Lønbro et al. [64] demonstrated an intergroup significant difference in favor of IG on QoL $(p<0.05)$ from baseline to week 12. Both studies used the same instrument (EORTC QLQ-C30). However, $\mathrm{O}^{\prime} \mathrm{Neill}$ et al. [65] did not show intergroup significant differences using the same instrument $(p=0.433)$. McNeely et al. [59] and Su et al. [63] measured QoL with FACT H\&N, and their results did not show any intergroup significant differences at any time point $(p>0.05)$. Additionally, McNeely et al. [58] did not show intergroup significant differences using FACT G. Concerning intragroup results, only Chang et al. [62] and Lønbro et al. [64] had favorable results for both the IG $(p<0.001)$ and CG $(p<0.05)$ postintervention. Considering studies that tested electrophysical agents, no intergroup differences were found $(p>0.05)$ [60,61,66,67]; however, the majority of the studies $[60,66,67]$ showed favorable intragroup results for both IG and CG at different time points $(p<0.05)$, although different instruments were used as outcomes (H\&N Cancer Inventory, OHIP-14, FACT H\&N). Regarding follow up, only three studies assessed cumulative effects $[61,62,65]$ as it was described previously, but none of the studies found effects maintained over time (Table 2).

\subsection{Risk of Bias in the Included RCTs}

The results of the methodological quality assessment of the 10 included RCTs are shown in Figure 2.

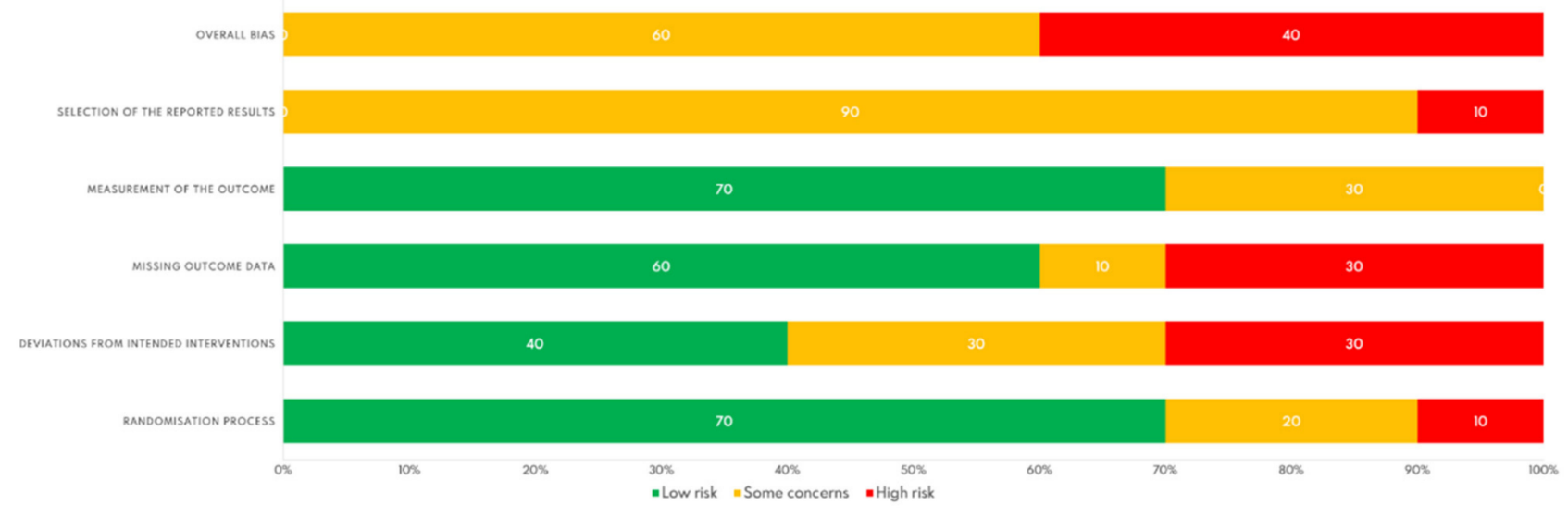

Figure 2. Risk of bias graph.

The major methodological quality issues were deviations from intended interventions $(30 \%)$ and missing outcome data (30\%) with "high risk". In contrast, "low risk" percentages were reported for the randomization process $(70 \%)$ and measurement of the outcome (70\%). Figure 3 shows an assessment summary for each study. All the studies included in this systematic review failed (partial or totally) in the selection of the reported result. Therefore, none of the studies achieved a "low" overall risk of bias. 


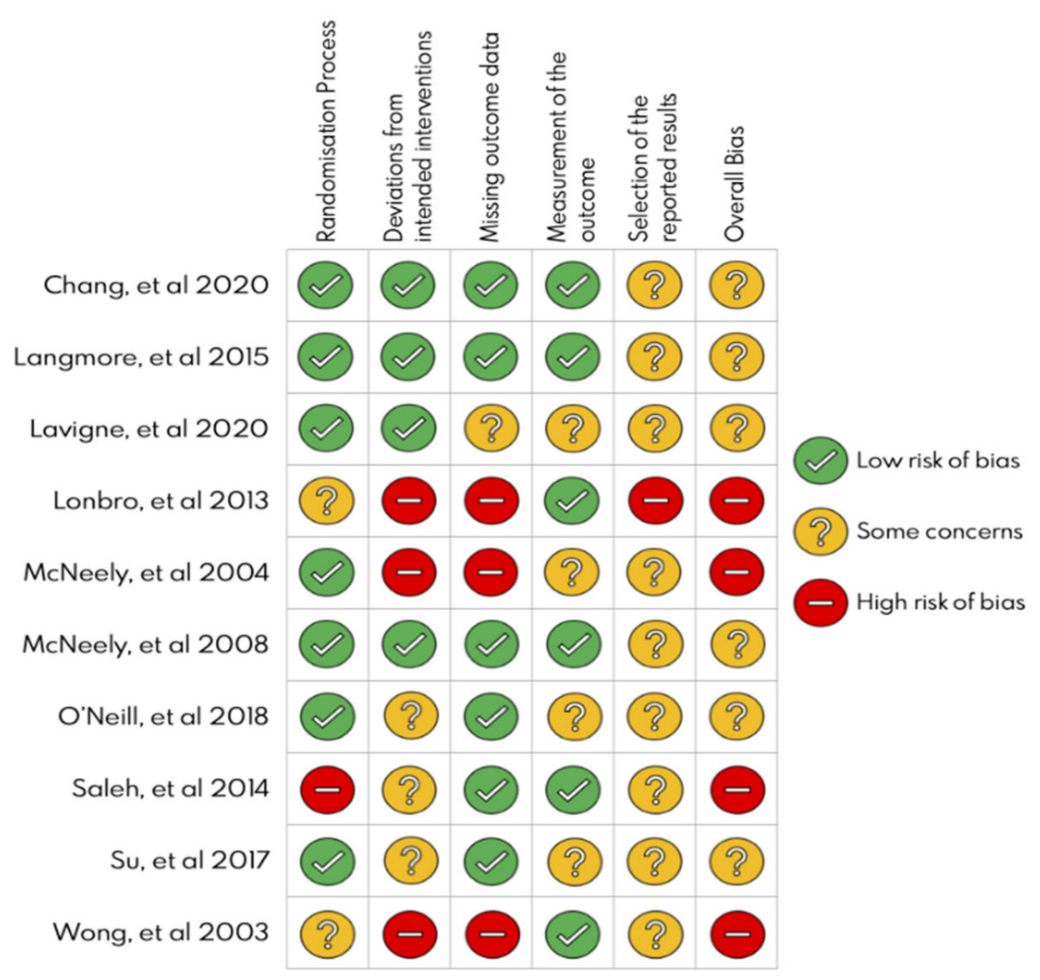

Figure 3. Risk of bias summary of the included studies.

\subsection{Meta-Analysis}

Of the ten studies included in the systematic review that measured QoL, it was only possible to include five in the meta-analysis [58,59,63-65]; the study by Chang et al. [62] was excluded, as it did not present the necessary data for extraction in the meta-analysis.

The meta-analysis included a total of 182 participants, 93 (IG) and 89 (CG). Regarding the assessment instruments used to measure QoL, two studies used the EORTC QLQC30 [64,65], two others used the FACT H\&N [59,63] and McNeely et al. [58] used the FACT G. McNeely et al.'s [59] study also measured QoL with the latter questionnaire, so its data were used for pooling. In the case of the EORTC QLQ-C30, the items are scored on a Likert scale of four points and have subscales (functioning, symptoms and global health). Higher scores on the functioning subscales and global health status reflect better health conditions; in contrast, higher scores on symptom subscales show critical symptoms, and finally, the subscale scores are transformed to a scale from 0 to 100 [52]. The FACT G has 27 items and also uses a Likert scale, but of five points; the subitems of this questionnaire are physical well-being, social/family well-being, emotional well-being and functional well-being. Higher scores mean better conditions, and by adding the subitems, the total score of the FACT G ranges from 0 to 108 points. The FACT H\&N has 39 items (27 of FACT $\mathrm{G}$ and 12 more of specific symptoms); on all of the instruments, higher scores are related to better QoL [53].

Regarding the data presented, there seems to be a tendency in favor of IG in terms of improvement in QoL after exercise program intervention (pooled Cohen's d 0.11; 95\% CI: -0.27 to $0.50 ; \mathrm{I}^{2} 42.68 \%$; $p$ heterogeneity $=0.12$ ). Pooled results are presented in Figure 4. 


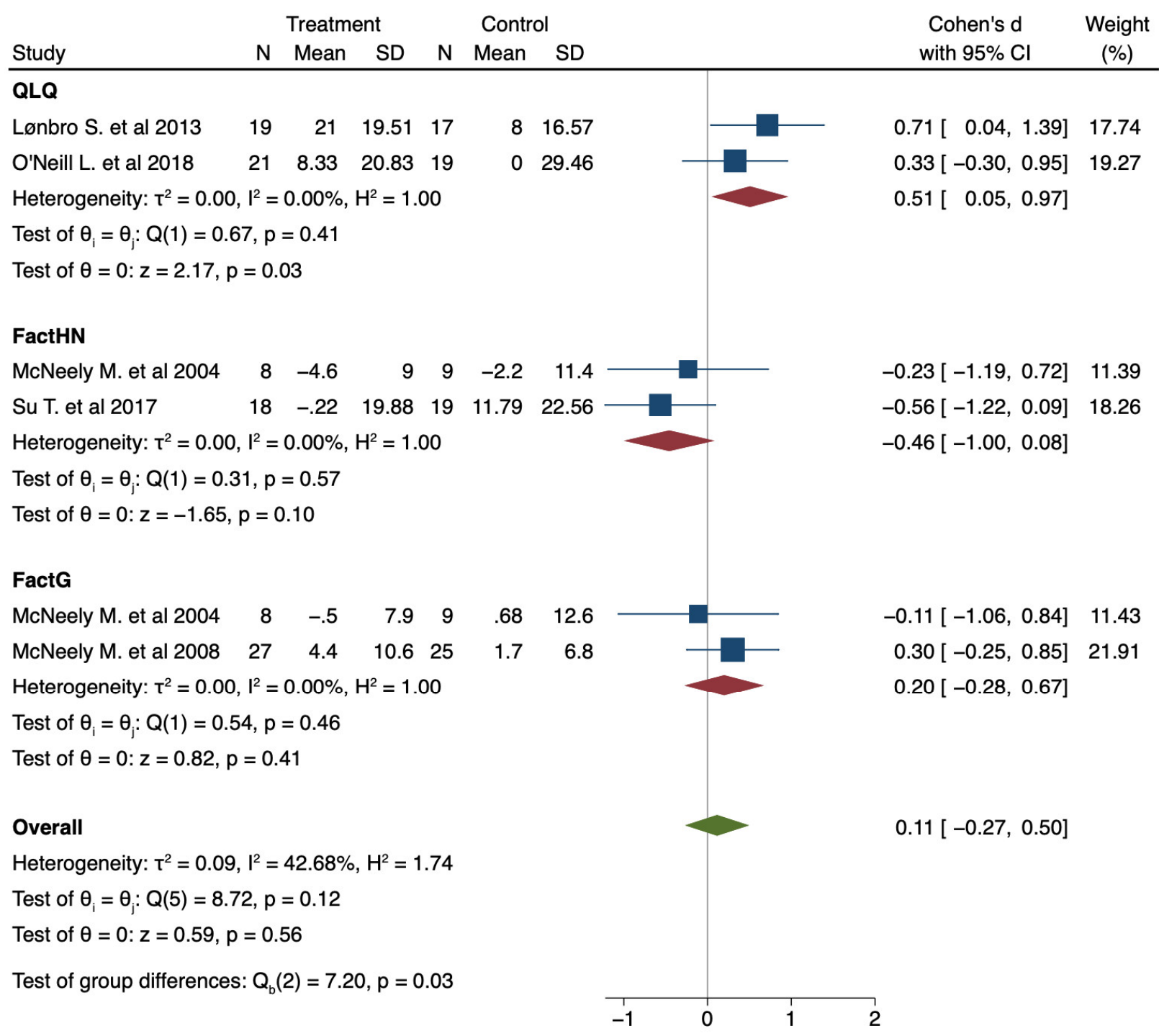

Figure 4. Forest plot presenting the effect of exercise on the improvement of Quality of Life (QoL) measured with different instruments in patients with Head and Neck cancer (HNC) compared with control; pre-post intervention data. Values on x-axis denote Cohen's d. The diamond illustrates the $95 \%$ confidence interval of the pooled effects.

\section{Discussion}

The main findings of this systematic review were that 12-week exercise programs focusing on aerobic activity (walking) or PRT for the whole body seem to be the modalities with more benefits to ameliorate QoL perception in HNC survivors. Neither electrophysical agent (electrical stimulation or laser therapy) showed significant results between groups, although almost every group improved their results. The meta-analysis supports the results in favor of exercise programs.

HNC survivors are recognized as a heterogeneous population in regard to the location of the tumor [9], and as a troublesome area of the body, it would be interesting to describe which programs involving aerobic and/or resistance exercises might be recommended $[62,64]$. The rest of the approaches did not detect differences in QoL, which could be related to the instrument used to measure QoL $[58,59,63,65]$; it is known that the EORTC QLQ-C30 is the most widely used multidimensional assessment of health-related quality of life [52]. Oneill et al. [65] stated that, although EORTC QLQ-C30 was used in their assessment, surprisingly, improvements were not found following their intervention. This instrument may not be the most suitable to detect subjective benefits described by their participants (e.g., confidence, social functioning). The findings defend the EORTC QLQ-C30 
as an overall instrument able to demonstrate the effect of different modalities of exercise, particularly in a population dealing with numerous treatment-related morbidities [7-13]. The rest of the instruments, which are presumably more specific (e.g., FACT H\&N) [53], may have been less sensitive to the effect of the mentioned approaches $[58,59,63]$. Exercises that involved the whole body have been used, and it seems logical to believe that changes in overall QoL would be desirable; hence, a global instrument such as the EORTC QLQ-C30 should be used in future studies. Additionally, there were differences between the studies of this review on frequency and intensity of the PRT [58,59,63-65]. Although guidelines on this matter are general for survivors of cancer $[68,69]$, parameters of doses/response could be a future line of research.

On the one hand, there are reports that involved only upper limb and scapular muscles [58,59,63], and the fact that Lønbro et al. [64] also involved spine muscles could have been decisive. All exercise programs described in this systematic review focused on strengthening the whole body (upper and lower limbs and even spine muscles), although the most popular locations in this review were the pharynx and oral cavity $[58,59,63,64]$. A possible explication could be that sensitive disorders caused by both cancer and treatment can determine this selection of global intervention instead of others more specifically $[7,70,71]$. Other low-intensity exercises whose target is the oral and cervical regions may have reported better results in terms of pain, which could translate to better QoL [7]. The potential mechanism responsible for these changes could be the increases of muscle mass, muscle strength and functional performance [68]. Although the search strategy was complex, other oral-cervical-based modalities, such as massage, were missed in this review if the majority of patients retrieved underwent a process of surgery that supposes an important physical cost, themselves [72-74].

Another detail that could have been crucial would be the use of an informatics component [62]. Monitoring compliance plus guidelines for diet and lifestyle changes are key challenges, particularly in survivors of cancer where habitual exercise participation is limited [75,76]. In contrast and, according to this review, an excellent adherence rate (approximately 93\%) was registered in all exercise programs [58,59,62-65]. A proposal for active telerehabilitation, based on feedback technology and face-to-face contact and designed with patients' perspectives in mind, would be more appropriate to involve patients in treatment $[77,78]$ and possibly reach a greater level of significance.

Finally, all reports were performed over 12 weeks (with or without significance), so it seems to be an adequate point to observe benefits in terms of QoL. Both modalities (aerobic and/or resistance exercises) should be considered in the rehabilitation of HNC survivors. Research within breast and colon cancer populations has already demonstrated relevant effects of different exercise interventions on global health status, pain, QoL and fatigue through clinical trials [79-84]. Additionally, the results are coincident with other reviews on breast and colon cancer patients [28-33], considering that this intervention should be initiated as soon as possible in the early posttreatment period, even from diagnosis, as the literature increasingly supports it $[25,85]$.

Regarding electrophysical agent studies, there was no benefit between groups, even when the intervention and comparison groups were successfully separated $[60,66,67]$. Several explanations could be suitable: basic oral hygiene given all patients [66] could be responsible for improvements due to the fact that, although its evidence is limited, it is known that a basic self-care protocol might achieve certain symptomatic relief [86]. Similar justification could be behind the improvement described by Lavigne et al. [60], as the use of an exercise CG within trial would justify benefits in both groups. Other work led by Langmore et al. [67] showed disappointing results on their main outcome (swallowing function); however, all their patients reported better QoL, which could be due to a simple placebo effect of participation in clinical trials [87]. Wong et al. [61] reported benefits in xerostomia symptoms throughout different protocols of ALTENS, but this did not result in better QoL. The authors suggest that a well-designed, placebo-controlled trial should be undertaken to further evaluate their hypothesis; however, some of these authors 
explained years later that appropriate sham control remains methodologically challenging for this modality [88]. Although three out of four studies reported the usage of electrical stimulation (neuromuscular and meridian-based points), the heterogeneity of parameters was decisive to complicate a consensus. A recent review with meta-analysis suggests that laser therapy is an effective, noninvasive and safe approach in patients with xerostomia (cancer and no cancer) [89]. However, the lack of significant results described by Saleh et al. [66] could have been related to the late effects of RT on glandular structure and its permanent damage over time [90] due to patients were treated at least 6 months after RT, where the potential benefits of laser could be lower.

Meta-analysis suggests that patients in the exercise group improved their QoL compared to the CG. Regarding the results and the aim of systematic review, the possibility of carrying out a network meta-analysis was raised to determine the mediation effect between the different physical therapy interventions proposed in the global effect on QoL and to establish the best possible treatment. However, although it would have been interesting to know a global estimator of the efficacy of these interventions in improving the QoL of HNC, the heterogeneity of these interventions, added to the small number of studies collected, made it impossible to combine them quantitatively. However, this same reasoning supports the results of the meta-analysis in favor of exercise: the inconclusive results of the other techniques and the low number of studies, together with the significant tendency shown in the meta-analysis, seem to postulate exercise as the gold standard in improving the QoL of these patients.

The results are in line with several meta-analyses [77,91-93] studying the effect of exercise in different cancer populations, mainly breast cancer, all of which showed results in favor of the intervention group. Finally, the low or null statistical heterogeneity observed in the subgroup analysis carried out is noteworthy, which reinforces the results of the meta-analysis, despite the small number of studies included. Although it is true that this heterogeneity is moderate in the overall analysis, this is explained by the variability of the assessment instrument used to measure QoL, which the choice of the statistical method used is intended to counteract.

To our knowledge, this is the first review evaluating the effect of different physical therapy modalities on improving QoL in HNC survivors. The strengths of this review are reporting according to the PRISMA guidelines, inclusion of risk of bias assessment and meta-analysis with the low statistical heterogeneity obtained.

\section{Study Limitations}

Limitations in published reports restricted the ability to determine those parameters of the interventions that were effective; further limitations include that the meta-analysis comprises only five studies and none of studies achieved a low overall risk of bias assessment, and it was not possible to combine the data from the studies that used electrophysical agents due to lack of methodological similarity between them.

\section{Conclusions}

In summary, this systematic review identified meaningful benefits of exercise with regard to QoL. This has been confirmed in a meta-analysis that comprises five out of ten studies involved. However, reports based on electrophysical agents such as electrostimulation and laser therapy were not able to find results between groups despite groups improving separately. The maintenance of effects in both modalities was also inconclusive. The potential of exercise and partly electrophysical agents to enhance QoL in HNC survivors seems to be clear, although it has not been possible to reach a reliable consensus in terms of the parameters analyzed due to the disparity in the data retrieved. This systematic review has brought to light the gaps in physical therapy strategies that affect this oncology population. This issue can be a starting point for future lines of research. To reinforce the emerging findings, it has found that all methods evaluated seem to be safe. This review adds to the growing evidence supporting exercise interventions to improve QoL in a cancer 
population such as HNC survivors, whose opportunities for successful recovery after medical treatment are more limited.

Author Contributions: Conceptualization, B.B.-M. and N.G.-C.; methodology, B.B.-M., M.L.-L. and N.G.-C.; formal analysis, M.L.-L., M.L.-G. and C.F.-L.; writing-original draft preparation, B.B.-M., M.L.-L., N.G.-C., C.F.-L. and M.L.-G.; writing-review and editing, B.B.-M., N.G.-C. and M.A.-M.; supervision, N.G.-C. and M.A.-M.; funding acquisition, N.G.-C. All authors have read and agreed to the published version of the manuscript.

Funding: This work was supported by the University of Granada: "Proyectos de Investigación Precompetitivos para Jóvenes Investigadores. Plan Propio 2020", Code: PPJIA2020.15.

Data Availability Statement: Not applicable.

Acknowledgments: This work was part of a Ph.D. thesis conducted in the Clinical Medicine and Public Health Doctoral Studies of the University of Granada, Spain.

Conflicts of Interest: The authors declare no conflict of interest.

\section{References}

1. Marur, S.; Forastiere, A.A. Head and Neck Squamous Cell Carcinoma: Update on Epidemiology, Diagnosis, and Treatment. Mayo Clin. Proc. 2016, 91, 386-396. [CrossRef]

2. Haddad, R.I.; Shin, D.M. Recent Advances in Head and Neck Cancer. N. Engl. J. Med. 2008, 359, 1143-1154. [CrossRef]

3. Haddad, R. Epidemiology, Staging, and Clinical Presentation of Human Papillomavirus-Associated Head and Neck Cancer. Available online: https: / / www.uptodate.com/contents/epidemiology-staging-and-clinical-presentation-of-human-papillomavirusassociated-head-and-neck-cancer?topicRef=89977\&source=see_link (accessed on 14 November 2020).

4. Howlader, N.; Noone, A.M.; Krapcho, M.; Miller, D.; Bishop, K.; Altekruse, S.F.; Kosary, C.L.; Yu, M.; Ruhl, J.; Tatalovich, Z.; et al. National Cancer Institute SEER Cancer Statistics Review 1975-2013. Available online: http://seer.cancer.gov/csr/1975_2013/ (accessed on 13 October 2021).

5. Cancer Research UK. Head and Neck Cancers Statistics. Available online: https://www.cancerresearchuk.org/healthprofessional/cancer-statistics/statistics-by-cancer-type/head-and-neck-cancers\#heading-Two (accessed on 17 June 2020).

6. Wissinger, E.; Griebsch, I.; Lungershausen, J.; Foster, T.; Pashos, C.L. The Economic Burden of Head and Neck Cancer: A Systematic Literature Review. Pharmacoeconomics 2014, 32, 865-882. [CrossRef]

7. Ortiz-Comino, L.; Fernández-Lao, C.; Castro-Martín, E.; Lozano-Lozano, M.; Cantarero-Villanueva, I.; Arroyo-Morales, M.; Martín-Martín, L. Myofascial Pain, Widespread Pressure Hypersensitivity, and Hyperalgesia in the Face, Neck, and Shoulder Regions, in Survivors of Head and Neck Cancer. Support. Care Cancer 2020, 28, 2891-2898. [CrossRef]

8. Bozec, A.; Schultz, P.; Gal, J.; Chamorey, E.; Chateau, Y.; Dassonville, O.; Poissonnet, G.; Demard, F.; Peyrade, F.; Saada, E.; et al. Evolution and Predictive Factors of Quality of Life in Patients Undergoing Oncologic Surgery for Head and Neck Cancer: A Prospective Multicentric Study. Surg. Oncol. 2019, 28, 236-242. [CrossRef]

9. Nayak, S.G.; Pai, M.S.; George, S.L. Quality of Life of Patients with Head and Neck Cancer. J. Cancer Res. Ther. 2019, 15, 638-644. [CrossRef] [PubMed]

10. Bhandari, S.; Soni, B.W.; Bahl, A.; Ghoshal, S. Radiotherapy-Induced Oral Morbidities in Head and Neck Cancer Patients. Spec. Care Dentist 2020, 40, 238-250. [CrossRef] [PubMed]

11. McDowell, L.J.; Rock, K.; Xu, W.; Chan, B.; Waldron, J.; Lu, L.; Ezzat, S.; Pothier, D.; Bernstein, L.J.; So, N.; et al. Long-Term Late Toxicity, Quality of Life, and Emotional Distress in Patients with Nasopharyngeal Carcinoma Treated With Intensity Modulated Radiation Therapy. Int. J. Radiat. Oncol. Biol. Phys. 2018, 102, 340-352. [CrossRef]

12. Huang, J.; Zhang, J.; Shi, C.; Liu, L.; Wei, Y. Survival, Recurrence and Toxicity of HNSCC in Comparison of a Radiotherapy Combination with Cisplatin versus Cetuximab: A Meta-Analysis. BMC Cancer 2016, 16. [CrossRef] [PubMed]

13. Crowder, S.L.; Douglas, K.G.; Yanina Pepino, M.; Sarma, K.P.; Arthur, A.E. Nutrition Impact Symptoms and Associated Outcomes in Post-Chemoradiotherapy Head and Neck Cancer Survivors: A Systematic Review. J. Cancer Surviv. 2018, 12, 479-494. [CrossRef] [PubMed]

14. Parke, S.C.; Oza, S.; Shahpar, S.; Ngo-Huang, A.; Herbert, A.; Barksdale, T.; Gerber, L. Identifying Gaps in Research on Rehabilitation for Patients with Head and Neck Cancer: A Scoping Review. Arch. Phys. Med. Rehabil. 2019, 100, 2381-2388. [CrossRef]

15. Berry, L.L.; Davis, S.W.; Godfrey Flynn, A.; Landercasper, J.; Deming, K.A. Is It Time to Reconsider the Term “Cancer Survivor"? J. Psychosoc. Oncol. 2019, 37, 413-426. [CrossRef]

16. Surbone, A.; Annunziata, M.A.; Santoro, A.; Tirelli, U.; Tralongo, P. Cancer Patients and Survivors: Changing Words or Changing Culture? Ann. Oncol. 2013, 24, 2468-2471. [CrossRef]

17. Ringash, J.; Bernstein, L.J.; Devins, G.; Dunphy, C.; Giuliani, M.; Martino, R.; McEwen, S. Head and Neck Cancer Survivorship: Learning the Needs, Meeting the Needs. Semin. Radiat. Oncol. 2018, 28, 64-74. [CrossRef] 
18. Nilsen, M.L.; Mady, L.J.; Hodges, J.; Wasserman-Wincko, T.; Johnson, J.T. Burden of Treatment: Reported Outcomes in a Head and Neck Cancer Survivorship Clinic. Laryngoscope 2019, 129, E437-E444. [CrossRef] [PubMed]

19. Giuliani, M.; McQuestion, M.; Jones, J.; Papadakos, J.; Le, L.W.; Alkazaz, N.; Cheng, T.; Waldron, J.; Catton, P.; Ringash, J. Prevalence and Nature of Survivorship Needs in Patients with Head and Neck Cancer. Head Neck 2016, 38, 1097-1103. [CrossRef] [PubMed]

20. Giuliani, M.; Papadakos, J.; Broadhurst, M.; Jones, J.; McQuestion, M.; Le, L.W.; Beck, L.; Waldron, J.; Ringash, J. The Prevalence and Determinants of Return to Work in Head and Neck Cancer Survivors. Support. Care Cancer 2019, 27, 539-546. [CrossRef]

21. Haddad, R.I.; Limaye, S. Overview of Approach to Long-Term Survivors of Head and Neck Cancer. Available online: https:/ / www.uptodate.com/contents/overview-of-approach-to-long-term-survivors-of-head-and-neck-cancer (accessed on 14 November 2020).

22. Forsythe, L.P.; Alfano, C.M.; Leach, C.R.; Ganz, P.A.; Stefanek, M.E.; Rowland, J.H. Who Provides Psychosocial Follow-up Care for Post-Treatment Cancer Survivors? A Survey of Medical Oncologists and Primary Care Physicians. J. Clin. Oncol. 2012, 30, 2897-2905. [CrossRef]

23. Nekhlyudov, L.; Lacchetti, C.; Davis, N.B.; Garvey, T.Q.; Goldstein, D.P.; Nunnink, J.C.; Ruades Ninfea, J.I.; Salner, A.L.; Salz, T.; Siu, L.L. Head and Neck Cancer Survivorship Care Guideline: American Society of Clinical Oncology Clinical Practice Guideline Endorsement of the American Cancer Society Guideline. J. Clin. Oncol. 2017, 35, 1606-1621. [CrossRef]

24. Baldoman, D.; Vandenbrink, R. Physical Therapy Challenges in Head and Neck Cancer. Cancer Treat. Res. 2018, 174, 209-223. [CrossRef] [PubMed]

25. Galiano-Castillo, N.; Postigo-Martin, P.; Cantarero-Villanueva, I. The Role of Physical Therapists in Oncology: The Great Unknown. Phys. Ther. Rev. 2020, 25, 235-237. [CrossRef]

26. Gamble, G.L.; Gerber, L.H.; Spill, G.R.; Paul, K.L. The Future of Cancer Rehabilitation. Am. J. Phys. Med. Rehabil. 2011, 90, S76-S87. [CrossRef]

27. Isaksson, J.; Wilms, T.; Laurell, G.; Fransson, P.; Ehrsson, Y.T. Meaning of Work and the Process of Returning after Head and Neck Cancer. Support. Care Cancer 2016, 24, 205-213. [CrossRef] [PubMed]

28. Meneses-Echávez, J.F.; González-Jiménez, E.; Ramírez-Vélez, R. Effects of Supervised Exercise on Cancer-Related Fatigue in Breast Cancer Survivors: A Systematic Review and Meta-Analysis. BMC Cancer 2015, 15, 1-13. [CrossRef] [PubMed]

29. Soares Falcetta, F.; de Araújo Vianna Träsel, H.; de Almeida, F.K.; Rangel Ribeiro Falcetta, M.; Falavigna, M.; Dornelles Rosa, D. Effects of Physical Exercise after Treatment of Early Breast Cancer: Systematic Review and Meta-Analysis. Breast Cancer Res. Treat. 2018, 170, 455-476. [CrossRef]

30. Bekhet, A.H.; Abdalla, A.R.; Ismail, H.M.; Genena, D.M.; Osman, N.A.; El Khatib, A.; Abbas, R.L. Benefits of Aerobic Exercise for Breast Cancer Survivors: A Systematic Review of Randomized Controlled Trials. Asian Pac. J. Cancer Prev. 2019, 20, 3197-3209. [CrossRef] [PubMed]

31. Del-Rosal-Jurado, A.; Romero-Galisteo, R.; Trinidad-Fernández, M.; González-Sánchez, M.; Cuesta-Vargas, A.; Ruiz-Muñoz, M. Therapeutic Physical Exercise Post-Treatment in Breast Cancer: A Systematic Review of Clinical Practice Guidelines. J. Clin. Med. 2020, 9, 1239. [CrossRef]

32. Balhareth, A.; Aldossary, M.Y.; McNamara, D. Impact of Physical Activity and Diet on Colorectal Cancer Survivors' Quality of Life: A Systematic Review. World J. Surg. Oncol. 2019, 17, 1-12. [CrossRef]

33. Brandenbarg, D.; Korsten, J.H.W.M.; Berger, M.Y.; Berendsen, A.J. The Effect of Physical Activity on Fatigue among Survivors of Colorectal Cancer: A Systematic Review and Meta-Analysis. Support. Care Cancer 2018, 26, 393-403. [CrossRef]

34. Liu, L.; He, X.; Feng, L. Exercise on Quality of Life and Cancer-Related Fatigue for Lymphoma Survivors: A Systematic Review and Meta-Analysis. Support. Care Cancer 2019, 27, 4069-4082. [CrossRef]

35. Toohey, K.; Pumpa, K.; McKune, A.; Cooke, J.; Semple, S. High-Intensity Exercise Interventions in Cancer Survivors: A Systematic Review Exploring the Impact on Health Outcomes. J. Cancer Res. Clin. Oncol. 2018, 144. [CrossRef] [PubMed]

36. Fuller, J.T.; Hartland, M.C.; Maloney, L.T.; Davison, K. Therapeutic Effects of Aerobic and Resistance Exercises for Cancer Survivors: A Systematic Review of Meta-Analyses of Clinical Trials. Br. J. Sports Med. 2018, 52, 1311. [CrossRef]

37. Ma, S.J.; Rivers, C.I.; Serra, L.M.; Singh, A.K. Long-Term Outcomes of Interventions for Radiation-Induced Xerostomia: A Review. World J. Clin. Oncol. 2019, 10, 1-13. [CrossRef] [PubMed]

38. Paim, É.D.; Berbert, M.C.B.; Zanella, V.G.; Macagnan, F.E. Electrical Stimulation in the Treatment of Radiotherapy-Induced Hyposalivation. Codas 2019, 31, 1-7. [CrossRef]

39. Sousa, A.S.; Silva, J.F.; Pavesi, V.C.S.; Carvalho, N.A.; Ribeiro-Júnior, O.; Varellis, M.L.Z.; Prates, R.A.; Bussadori, S.K.; Gonçalves, M.L.L.; Horliana, A.C.R.T.; et al. Photobiomodulation and Salivary Glands: A Systematic Review. Lasers Med. Sci. 2020, 35, 777-788. [CrossRef] [PubMed]

40. Mercadante, V.; Al Hamad, A.; Lodi, G.; Porter, S.; Fedele, S. Interventions for the Management of Radiotherapy-Induced Xerostomia and Hyposalivation: A Systematic Review and Meta-Analysis. Oral Oncol. 2017, 66, 64-74. [CrossRef]

41. Shao, C.H.; Chiang, C.C.; Huang, T.W. Exercise Therapy for Cancer Treatment-Induced Trismus in Patients with Head and Neck Cancer: A Systematic Review and Meta-Analysis of Randomized Controlled Trials. Radiother. Oncol. 2020, 151, $249-255$. [CrossRef]

42. Almeida, K.A.M.; Rocha, A.P.; Carvas, N.; Pinto, A.C.P.N. Rehabilitation Interventions for Shoulder Dysfunction in Patients with Head and Neck Cancer: Systematic Review and Meta-Analysis. Phys. Ther. 2020, 100, 1997-2008. [CrossRef] 
43. Cousins, N.; MacAulay, F.; Lang, H.; MacGillivray, S.; Wells, M. A Systematic Review of Interventions for Eating and Drinking Problems Following Treatment for Head and Neck Cancer Suggests a Need to Look beyond Swallowing and Trismus. Oral Oncol. 2013, 49, 387-400. [CrossRef]

44. Heiskanen, V.; Zadik, Y.; Elad, S. Photobiomodulation Therapy for Cancer Treatment-Related Salivary Gland Dysfunction: A Systematic Review. Photobiomodulation Photomed. Laser Surg. 2020, 38, 340-347. [CrossRef]

45. Kamstra, J.I.; van Leeuwen, M.; Roodenburg, J.L.N.; Dijkstra, P.U. Exercise Therapy for Trismus Secondary to Head and Neck Cancer: A Systematic Review. Head Neck 2017, 39, 160-169. [CrossRef]

46. Kraaijenga, S.A.C.; Van Der Molen, L.; Van Den Brekel, M.W.M.; Hilgers, F.J.M. Current Assessment and Treatment Strategies of Dysphagia in Head and Neck Cancer Patients: A Systematic Review of the 2012/13 Literature. Curr. Opin. Support. Palliat. Care 2014, 8, 152-163. [CrossRef]

47. Perry, A.; Lee, S.H.; Cotton, S.; Kennedy, C. Therapeutic Exercises for Affecting Post-Treatment Swallowing in People Treated for Advanced-Stage Head and Neck Cancers. Cochrane Database Syst. Rev. 2016, 2016. [CrossRef] [PubMed]

48. Scherpenhuizen, A.; Van Waes, A.M.A.; Janssen, L.M.; Van Cann, E.M.; Stegeman, I. The Effect of Exercise Therapy in Head and Neck Cancer Patients in the Treatment of Radiotherapy-Induced Trismus: A Systematic Review. Oral Oncol. 2015, 51, 745-750. [CrossRef] [PubMed]

49. Tyker, A.; Franco, J.; Massa, S.T.; Desai, S.C.; Walen, S.G. Treatment for Lymphedema Following Head and Neck Cancer Therapy: A Systematic Review. Am. J. Otolaryngol. Head Neck Med. Surg. 2019, 40, 761-769. [CrossRef] [PubMed]

50. Moher, D.; Liberati, A.; Tetzlaff, J.; Altman, D.G.; Altman, D.; Antes, G.; Atkins, D.; Barbour, V.; Barrowman, N.; Berlin, J.A.; et al. Preferred Reporting Items for Systematic Reviews and Meta-Analyses: The PRISMA Statement. PLoS Med. 2009, 6. [CrossRef]

51. Veritas Health Innovation. Covidence Systematic Review Software. Available online: www.covidence.org (accessed on 13 October 2021).

52. Bjordal, K.; Graeff, A.; Fayers, P.M.; Hammerlid, E.; Pottelsberghe, C.V.; Arraras, J.J.; Feine, J.S.; Abendstein, H.; Morton, R.P. A 12 Country Eld Study of the EORTC QLQ-C30 (Version 3.0) and the Head and Neck Cancer Specific Module (EORTC QLQ-H\&N35) in Head and Neck Patients. EORTC Quality of Life Group. Eur. J. Cancer 2000, 36, 1796-1807.

53. Yount, S.; List, M.; Du, H.; Yost, K.; Bode, R.; Brockstein, B.; Argiris, A.; Vokes, E.; Cohen, E.E.W.; Campbell, B.; et al. A Randomized Validation Study Comparing Embedded versus Extracted FACT Head and Neck Symptom Index Scores. Qual. Life Res. 2007, 16, 1615-1626. [CrossRef]

54. Funk, G.F.; Karnell, L.H.; Christensen, A.J.; Moran, P.J.; Ricks, J. Comprehensive Head and Neck Oncology Health Status Assessment. Head Neck 2003, 25, 561-575. [CrossRef] [PubMed]

55. Sterne, J.A.C.; Savović, J.; Page, M.J.; Elbers, R.G.; Blencowe, N.S.; Boutron, I.; Cates, C.J.; Cheng, H.Y.; Corbett, M.S.; Eldridge, S.M.; et al. RoB 2: A Revised Tool for Assessing Risk of Bias in Randomised Trials. BMJ 2019, 366, 1-8. [CrossRef]

56. Higgins, J.P.T. Measuring Inconsistency in Meta-Analyses. BMJ 2003, 327, 557-560. [CrossRef]

57. Landis, J.R.; Koch, G.G. Landis Amd Koch1977_agreement of Categorical Data. Biometrics 1977, 33, 159-174. [CrossRef]

58. McNeely, M.L.; Parliament, M.B.; Seikaly, H.; Jha, N.; Magee, D.J.; Haykowsky, M.J.; Courneya, K.S. Effect of Exercise on Upper Extremity Pain and Dysfunction in Head and Neck Cancer Survivors: A Randomized Controlled Trial. Cancer 2008, 113, $214-222$. [CrossRef]

59. McNeely, M.L.; Parliament, M.; Courneya, K.S.; Seikaly, H.; Jha, N.; Scrimger, R.; Henson, J. A Pilot Study of a Randomized Controlled Trial to Evaluate the Effects of Progressive Resistance Exercise Training on Shoulder Dysfunction Caused by Spinal Accessory Neurapraxia/Neurectomy in Head and Neck Cancer Survivors. Head Neck 2004, 26, 518-530. [CrossRef]

60. Lavigne, C.; Twomey, R.; Lau, H.; Francis, G.; Culos-Reed, S.N.; Millet, G.Y. Feasibility of Eccentric Overloading and Neuromuscular Electrical Stimulation to Improve Muscle Strength and Muscle Mass after Treatment for Head and Neck Cancer. J. Cancer Surviv. 2020, 14, 790-805. [CrossRef]

61. Wong, R.K.W.; Jones, G.W.; Sagar, S.M.; Babjak, A.F.; Whelan, T. A Phase I-II Study in the Use of Acupuncture-like Transcutaneous Nerve Stimulation in the Treatment of Radiation-Induced Xerostomia in Head-and-Neck Cancer Patients Treated with Radical Radiotherapy. Int. J. Radiat. Oncol. Biol. Phys. 2003, 57, 472-480. [CrossRef]

62. Chang, Y.L.; Tsai, Y.F.; Hsu, C.L.; Chao, Y.K.; Hsu, C.C.; Lin, K.C. The Effectiveness of a Nurse-Led Exercise and Health Education Informatics Program on Exercise Capacity and Quality of Life among Cancer Survivors after Esophagectomy: A Randomized Controlled Trial. Int. J. Nurs. Stud. 2020, 101, 103418. [CrossRef]

63. Su, T.L.; Chen, A.N.; Leong, C.P.; Huang, Y.C.; Chiang, C.W.; Chen, I.H.; Lee, Y.Y. The Effect of Home-Based Program and Outpatient Physical Therapy in Patients with Head and Neck Cancer: A Randomized, Controlled Trial. Oral Oncol. 2017, 74, 130-134. [CrossRef] [PubMed]

64. Lønbro, S.; Dalgas, U.; Primdahl, H.; Johansen, J.; Nielsen, J.L.; Aagaard, P.; Hermann, A.P.; Overgaard, J.; Overgaard, K. Progressive Resistance Training Rebuilds Lean Body Mass in Head and Neck Cancer Patients after Radiotherapy-Results from the Randomized DAHANCA 25B Trial. Radiother. Oncol. 2013, 108, 314-319. [CrossRef] [PubMed]

65. O'Neill, L.M.; Guinan, E.; Doyle, S.L.; Bennett, A.E.; Murphy, C.; Elliott, J.A.; O'Sullivan, J.; Reynolds, J.V.; Hussey, J. The RESTORE Randomized Controlled Trial: Impact of a Multidisciplinary Rehabilitative Program on Cardiorespiratory Fitness in Esophagogastric Cancer Survivorship. Ann. Surg. 2018, 268, 747-755. [CrossRef] [PubMed]

66. Saleh, J.; Figueiredo, M.A.Z.; Cherubini, K.; Braga-Filho, A.; Salum, F.G. Effect of Low-Level Laser Therapy on RadiotherapyInduced Hyposalivation and Xerostomia: A Pilot Study. Photomed. Laser Surg. 2014, 32, 546-552. [CrossRef] 
67. Langmore, S.E.; McCulloch, T.M.; Krisciunas, G.P.; Lazarus, C.L.; Van Daele, D.J.; Pauloski, B.R.; Rybin, D.; Doros, G. Efficacy of Electrical Stimulation and Exercise for Dysphagia in Patients with Head and Neck Cancer: A Randomized Clinical Trial. Head Neck 2016, 38, E1221-E1231. [CrossRef] [PubMed]

68. Schmitz, K.H.; Courneya, K.S.; Matthews, C.; Demark-Wahnefried, W.; Galvão, D.A.; Pinto, B.M.; Irwin, M.L.; Wolin, K.Y.; Segal, R.J.; Lucia, A.; et al. American College of Sports Medicine Roundtable on Exercise Guidelines for Cancer Survivors. Med. Sci. Sports Exerc. 2010, 42, 1409-1426. [CrossRef]

69. Services, H. Physical Activity Guidelines Advisory Committee Report, 2008: To the Secretary of Health and Human Services. Nutr. Rev. 2009, 67, 114-120. [CrossRef]

70. Stubblefield, M.D. Radiation Fibrosis Syndrome: Neuromuscular and Musculoskeletal Complications in Cancer Survivors. PMER 2011, 3, 1041-1054. [CrossRef]

71. Van Wilgen, C.P.; Dijkstra, P.U.; van der Laan, B.F.A.M.; Plukker, J.T.; Roodenburg, J.L.N. Morbidity of the Neck after Head and Neck Cancer Therapy. Head Neck 2004, 26, 785-791. [CrossRef]

72. Carr, S.D.; Bowyer, D.; Cox, G. Upper Limb Dysfunction Following Selective Neck Dissection: A Retrospective Questionnaire Study. Head Neck 2009, 31, 789-792. [CrossRef] [PubMed]

73. Gane, E.M.; O'Leary, S.P.; Hatton, A.L.; Panizza, B.J.; McPhail, S.M. Neck and Upper Limb Dysfunction in Patients Following Neck Dissection: Looking beyond the Shoulder. Otolaryngol. Neck Surg. 2017, 157, 631-640. [CrossRef]

74. Gane, E.M.; Michaleff, Z.A.; Cottrell, M.A.; McPhail, S.M.; Hatton, A.L.; Panizza, B.J.; O'Leary, S.P. Prevalence, Incidence, and Risk Factors for Shoulder and Neck Dysfunction after Neck Dissection: A Systematic Review. Eur. J. Surg. Oncol. 2017, 43, 1199-1218. [CrossRef] [PubMed]

75. Rock, C.L.; Doyle, C.; Demark-Wahnefried, W.; Meyerhardt, J.; Courneya, K.S.; Schwartz, A.L.; Bandera, E.V.; Hamilton, K.K.; Grant, B.; McCullough, M.; et al. Nutrition and Physical Activity Guidelines for Cancer Survivors. CA. Cancer J. Clin. 2012, 62, 242-274. [CrossRef] [PubMed]

76. Wu, Y.P.; Yi, J.; McClellan, J.; Kim, J.; Tian, T.; Grahmann, B.; Kirchhoff, A.C.; Holton, A.; Wright, J. Barriers and Facilitators of Healthy Diet and Exercise Among Adolescent and Young Adult Cancer Survivors: Implications for Behavioral Interventions. J. Adolesc. Young Adult Oncol. 2015, 4, 184-191. [CrossRef] [PubMed]

77. Cranen, K.; Groothuis-Oudshoorn, C.G.; Vollenbroek-Hutten, M.M.; IJzerman, M.J. Toward Patient-Centered Telerehabilitation Design: Understanding Chronic Pain Patients' Preferences for Web-Based Exercise Telerehabilitation Using a Discrete Choice Experiment. J. Med. Internet Res. 2017, 19, e26. [CrossRef] [PubMed]

78. Batalik, L.; Winnige, P.; Dosbaba, F.; Vlazna, D.; Janikova, A. Home-Based Aerobic and Resistance Exercise Interventions in Cancer Patients and Survivors: A Systematic Review. Cancers 2021, 13, 1915. [CrossRef]

79. Galiano-Castillo, N.; Cantarero-Villanueva, I.; Fernández-Lao, C.; Ariza-García, A.; Díaz-Rodríguez, L.; Del-Moral-Ávila, R.; Arroyo-Morales, M. Telehealth System: A Randomized Controlled Trial Evaluating the Impact of an Internet-Based Exercise Intervention on Quality of Life, Pain, Muscle Strength, and Fatigue in Breast Cancer Survivors. Cancer 2016, 122, 3166-3174. [CrossRef] [PubMed]

80. Dieli-Conwright, C.M.; Courneya, K.S.; Demark-Wahnefried, W.; Sami, N.; Lee, K.; Sweeney, F.C.; Stewart, C.; Buchanan, T.A.; Spicer, D.; Tripathy, D.; et al. Aerobic and Resistance Exercise Improves Physical Fitness, Bone Health, and Quality of Life in Overweight and Obese Breast Cancer Survivors: A Randomized Controlled Trial. Breast Cancer Res. 2018, 20, 124. [CrossRef] [PubMed]

81. Penttinen, H.; Utriainen, M.; Kellokumpu-Lehtinen, P.-L.; Raitanen, J.; Sievänen, H.; Nikander, R.; Blomqvist, C.; Huovinen, R.; Vehmanen, L.; Saarto, T. Effectiveness of a 12-Month Exercise Intervention on Physical Activity and Quality of Life of Breast Cancer Survivors; Five-Year Results of the BREX-Study. In Vivo 2019, 33, 881-888. [CrossRef]

82. Kim, J.Y.; Lee, M.K.; Lee, D.H.; Kang, D.W.; Min, J.H.; Lee, J.W.; Chu, S.H.; Cho, M.S.; Kim, N.K.; Jeon, J.Y. Effects of a 12-Week Home-Based Exercise Program on Quality of Life, Psychological Health, and the Level of Physical Activity in Colorectal Cancer Survivors: A Randomized Controlled Trial. Support. Care Cancer 2019, 27, 2933-2940. [CrossRef]

83. Brown, J.C.; Damjanov, N.; Courneya, K.S.; Troxel, A.B.; Zemel, B.S.; Rickels, M.R.; Ky, B.; Rhim, A.D.; Rustgi, A.K.; Schmitz, K.H. A Randomized Dose-Response Trial of Aerobic Exercise and Health-Related Quality of Life in Colon Cancer Survivors. Psychooncology 2018, 27, 1221-1228. [CrossRef]

84. Cantarero-Villanueva, I.; Cuesta-Vargas, A.I.; Lozano-Lozano, M.; Fernández-Lao, C.; Fernández-Pérez, A.; Galiano-Castillo, N. Changes in Pain and Muscle Architecture in Colon Cancer Survivors After a Lumbopelvic Exercise Program: A Secondary Analysis of a Randomized Controlled Trial. Pain Med. 2017, 18, 1366-1376. [CrossRef]

85. Postigo-Martin, P.; Peñafiel-Burkhardt, R.; Gallart-Aragón, T.; Alcaide-Lucena, M.; Artacho-Cordón, F.; Galiano-Castillo, N.; Fernández-Lao, C.; Martín-Martín, L.; Lozano-Lozano, M.; Ruíz-Vozmediano, J.; et al. Attenuating Treatment-Related Cardiotoxicity in Women Recently Diagnosed With Breast Cancer via a Tailored Therapeutic Exercise Program: Protocol of the ATOPE Trial. Phys. Ther. 2021, 101. [CrossRef]

86. Furness, S.; Worthington, H.V.; Bryan, G.; Birchenough, S.; McMillan, R. Interventions for the Management of Dry Mouth: Topical Therapies. Cochrane Database Syst. Rev. 2011, 12, CD008934. [CrossRef]

87. Doering, B.K.; Rief, W.; Petrie, K.J. Lessons to Be Learned from Placebo Arms in Psychopharmacology Trials. In Placebo; Springer: Heidelberg, Germany, 2014; pp. 273-290. 
88. Wong, R.K.W.; James, J.L.; Sagar, S.; Wyatt, G.; Nguyen-Tân, P.F.; Singh, A.K.; Lukaszczyk, B.; Cardinale, F.; Yeh, A.M.; Berk, L. Phase 2 Results from Radiation Therapy Oncology Group Study 0537. Cancer 2012, 118, 4244-4252. [CrossRef]

89. Galiano-Castillo, N.; Liu, L.; Lozano-Lozano, M.; Tumilty, S.; Cantarero-Villanueva, I.; Baxter, G.D. Acute and Cumulative Benefits of Photobiomodulation for Xerostomia: A Systematic Review and Meta-analysis. Oral Dis. 2021, 27, 1115-1126. [CrossRef]

90. Shiboski, C.H.; Hodgson, T.A.; Ship, J.A.; Schiødt, M. Management of Salivary Hypofunction during and after Radiotherapy. Oral Surgery, Oral Med. Oral Pathol. Oral Radiol. Endodontology 2007, 103, S66.e1-S66.e19. [CrossRef]

91. Cramp, F.; James, A.; Lambert, J. The Effects of Resistance Training on Quality of Life in Cancer: A Systematic Literature Review and Meta-Analysis. Support. Care Cancer 2010, 18, 1367-1376. [CrossRef] [PubMed]

92. McNeely, M.L. Effects of Exercise on Breast Cancer Patients and Survivors: A Systematic Review and Meta-Analysis. Can. Med. Assoc. J. 2006, 175, 34-41. [CrossRef] [PubMed]

93. Nadler, M.B.; Desnoyers, A.; Langelier, D.M.; Amir, E. The Effect of Exercise on Quality of Life, Fatigue, Physical Function, and Safety in Advanced Solid Tumor Cancers: A Meta-Analysis of Randomized Control Trials. J. Pain Symptom Manag. 2019, 58, 899-908. [CrossRef] [PubMed] 